\title{
A Pesquisa em Epidemiologia
}

A pesquisa no Brasil vem sendo impulsionada nas últimas décadas como resultado de políticas que, efetivamente, criaram oportunidades de formação de pesquisadores em grandes centros científicos em todo o mundo, incentivaram a consolidação de programas nacionais de formação em nível de excelência e a implantação de linhas de financiamento em áreas de interesse estratégico. No campo da saúde pública essa expansão tem se refletido, em especial, no crescimento acelerado da pesquisa epidemiológica. Estudos publicados recentemente têm documentado esta tendência e apresentado alguns aspectos relacionados a este crescimento. Utilizando a base de dados do Diretório de Grupos de Pesquisa do CNPq, Guimarães et al. (2001) mostraram que, em 2000, havia no país 363 pesquisadores com título de doutor, realizando pesquisa epidemiológica, estando organizados em torno de 176 grupos e 320 linhas de pesquisas. Destes, 94 (25,8\%) tinham obtido o título em universidades estrangeiras, principalmente européias ou americanas. Uma informação interessante é que, embora a maioria desses grupos (64\%) se concentrasse na área de Saúde Coletiva, sendo de apenas $14 \%$ a representação das demais áreas da Saúde, os restantes (22\%) estavam distribuídos por subáreas tão diversas quanto biomedicina, ecologia, física ou química. Assim, pode-se dizer que o crescimento da pesquisa em saúde no Brasil tem se concentrado no campo da Saúde Coletiva, com a epidemiologia sendo uma das principais abordagens metodológicas empregadas.

Esse crescimento da pesquisa em saúde no Brasil vem repercutindo na contribuição relativa da ciência brasileira na produção do conhecimento na América Latina e no mundo. Em um estudo sobre publicações científicas em saúde no período entre $1973 \mathrm{e}$ 1992, dentre os seis países de maior importância na pesquisa da América Latina, identificou-se que a produção científica brasileira era relevante e de impacto internacional (Pelegrini et al., 1997). Além disso, verificou-se que essa produção privilegiava o campo da Saúde Pública, conforme está evidente no fato de que, embora na Clínica e na Biomedicina a contribuição do Brasil e da Argentina, conjuntamente, fosse de 68 e 54\%, respectivamente, na Saúde Pública o Brasil sozinho respondia por $61 \%$ da produção. Como os autores analisaram apenas os trabalhos indexados no Institute of Scientific Information (ISI), onde grande parte dos trabalhos classificados como de Saúde Pública se concentra no campo da epidemiologia, isso demonstra que a produção brasileira em epidemiologia vem ocupando um espaço significativo em veículos de circulação internacional. 
Um outro aspecto interessante a se considerar na análise da pesquisa epidemiológica no Brasil é a sua orientação para os problemas de saúde de grande importância social, e a sua compreensão, construída a partir do suposto do papel central que ocupam os determinantes políticos, sociais, econômicos e culturais. Em uma ampla revisão da literatura sobre determinantes sociais em saúde, no período de 1980 a 2000, na qual utilizaram diversas fontes de informações bibliográficas, Almeida-Filho et al., (2003) demonstraram o crescimento exponencial de publicações relacionadas às pesquisas sobre esta temática na América Latina, e que o Brasil era responsável por aproximadamente metade delas.

Esta presença brasileira na ciência da saúde não se restringe apenas à área da América Latina. Em recente análise das publicações em epidemiologia registradas no Medline entre 1985 e 2004, Barreto (2005) observou que: a) o número de artigos aumentou cerca de 12 vezes, entre o qüinqüênio 1985-1989 e 2000-2004; b) a produção brasileira no primeiro período analisado, $1985-1989$, representava $0,6 \%$ por cento da produção mundial, aumentando para 1,2\% no último período, 2000-2004, o que significou um crescimento duas vezes maior do que o crescimento médio mundial; c) a proporção de artigos publicados no Brasil ou em língua inglesa, no Brasil e no exterior, manteve-se em níveis aproximadamente constantes em todo o período, em torno de $50 \%$ e $70 \%$, respectivamente. Em resumo, o padrão de crescimento da produção científica brasileira em epidemiologia não tem se diferenciado do observado para a ciência brasileira em sua globalidade (Guimarães, 2004). Vale ressaltar que é ainda grande o potencial de aumento da produção nacional em epidemiologia, haja vista a criação de novos programas de pós-graduação e o resultante aumento do número de dissertações e teses que necessitam de estímulo para a sua divulgação em periódicos indexados, que garantam a sua visibilidade para o grande público.

Existem evidências também de que o foco dessas pesquisas tem se voltado para questões de grande relevância e impacto potencial para a melhoria das condições de saúde da nossa população e dos serviços de saúde. Um exemplo disso é o desenvolvimento de pesquisas avaliativas de interesse estratégico, como a efetividade de vacinas e programas de grande abrangência, como o Programa de Saúde da Família, o Programa de Alimentação do Trabalhador, entre outros, ou ainda as pesquisas sobre determinantes sociais da saúde e desenvolvimento de crianças, a AIDS, ou sobre a violência urbana e doméstica, entre outros problemas que têm despertado o interesse da Saúde Pública mais recentemente.

O impacto deste crescimento sobre as práticas de saúde é de muito mais difícil identificação, e são ainda raros os estudos voltados para a análise da incorporação do conhecimento produzido nas políticas e práticas de saúde. Todavia, existem evidências 
de uma profunda e antiga relação entre os diversos organismos que compõem o SUS, em seus distintos níveis, e os grupos que realizam pesquisas epidemiológicas, especialmente em algumas áreas do conhecimento. Há também um claro intercâmbio de profissionais que ora se encontram no ambiente acadêmico, ora em posições administrativas na gestão dos serviços e instituições, o que possivelmente facilita o reconhecimento da importância e a incorporação da pesquisa na formulação de políticas. Ademais, profissionais de saúde vêm desenvolvendo as suas próprias pesquisas com os dados dos serviços, e ampliando de modo exponencial a sua participação em espaços tradicionalmente acadêmicos, como os congressos científicos e em especial os de epidemiologia. Isso tem possibilitado a criação de um ambiente adequado para que os problemas que emergem da prática cotidiana dos serviços de saúde sejam incorporados à pesquisa e, em sentido oposto, os resultados das investigações sejam empregados para a adequação das políticas e práticas dos serviços de saúde.

Os fatores responsáveis por este desenvolvimento são complexos, mas sem dúvida, o papel da ABRASCO e, em especial, da sua Comissão de Epidemiologia tem sido de grande relevância em apoio a esse processo. Ao se destacar da Saúde Coletiva com a criação de uma comissão específica em 1984, foi fortalecida a criação de uma comunidade científica no campo da epidemiologia, que passou a reconhecer as suas perspectivas, potencialidades e compromissos com a transformação das práticas de saúde, destacando-se, nesse movimento, o papel estratégico de uma pesquisa nacional enraizada nas necessidades de saúde do país. Foi esse reconhecimento que levou à elaboração dos três Planos Diretores para o Desenvolvimento da Epidemiologia no Brasil, identificados como importantes instrumentos de planejamento estratégico para a consolidação e constante aperfeiçoamento da prática de pesquisa epidemiológica no país.

\section{Referências}

Almeida Filho N, Kawachi I, Filho AP, Dachs JN. Research on health inequalities in Latin America and the Caribbean: bibliometric analysis (1971-2000) and descriptive content analysis (1971-1995). Am J Public Health 2003; 93(12): 2037-43.

Barreto ML. A pesquisa em epidemiologia no Brasil entre 1985-2004. Instituto de Saúde Coletiva, Universidade Federal da Bahia 2005. (Trabalho inédito).

Guimarães JA. A pesquisa médica e biomédica no Brasil. Comparações com o desempenho científico brasileiro e mundial. Ciência \& Saúde Coletiva 2004; 9(2): 303-27.

Guimarães R, Lourenço de Oliveira R, Cosac S. Epidemiological research in Brazil. Rev Saúde Pública 2001; 35(4): 321-40.

Pellegrini Filho A, Goldbaum M, Silvi J. Production of scientific articles about health in six Latin American countries, 1973-1992 . Rev Panam Salud Publica. 1997;1(1):23-34. 


\section{A produção do conhecimento em Epidemiologia}

\subsection{Problemas identificados}

1.1.1 Insuficiente articulação da pesquisa epidemiológica, especialmente em algumas áreas do conhecimento em saúde, com a política nacional de ciência e tecnologia e inovação;

1.1.2 Carência de fluxos estáveis e em montante adequado às necessidades de financiamento para a pesquisa epidemiológica;

1.1.3 Insuficiente articulação de áreas do conhecimento focalizadas na pesquisa em uma perspectiva inter ou transdisciplinar;

1.1.4 Insuficientes mecanismos de fixação de novos pesquisadores em instituições de algumas regiões brasileiras, e a lentidão da sua incorporação em grupos de pesquisa já existentes, o que pode contribuir para reduzir a lacuna de produção de conhecimento em saúde, especialmente na região norte;

1.1.5 Incipiência na formação de redes nacionais e internacionais de pesquisadores necessárias para dar respostas a questões complexas de investigação ou para o fortalecimento entre diferentes grupos de pesquisa;

1.1.6 Tendência de especialização do campo, com o seu conseqüente isolamento disciplinar, tanto ao interior do campo da Saúde Coletiva, como também com outras disciplinas da área maior da saúde.

\subsection{Ações propostas}

1.2.1 Ampliar a participação da comunidade nas instâncias de formulação e deliberação da política científica, tanto no âmbito nacional quanto no âmbito estadual;

1.2.2 Apoiar a implementação da Política Nacional de Ciência, Tecnologia e Inovação em Saúde e da Agenda de Prioridades em Pesquisa e Saúde, aprovadas pela $2^{\text {a }}$ Conferência Nacional de Ciência, Tecnologia e Inovação em Saúde;

1.2.3 Buscar maior participação da comunidade científica nas agências de formulação, deliberação e implementação da política científica em todas as instân- 
cias, com especial ênfase para as Fundações Estaduais de Amparo à Pesquisa e, em particular, se articular politicamente com vistas ao cumprimento da legislação relativa ao repasse de percentual do orçamento estadual;

1.2.4 Apoiar a criação de um órgão de fomento à pesquisa em saúde, vinculado ao Ministério da Saúde, com vistas a garantir a regularidade no financiamento, a desburocratização e eficiência nos procedimentos de repasse, bem como a transparência nos processos de seleção e contratação de projetos;

1.2.5 Incentivar e colaborar na elaboração de editais que contemplem de maneira prioritária os problemas de saúde de maior relevância nacional, com consideração especial para a complexidade de algumas dessas temáticas, estimulando a pesquisa inter e transdisciplinar e a formação de redes;

1.2.6 Propiciar oportunidades para o encontro de pesquisadores e grupos de pesquisa em áreas temáticas específicas, com vistas ao incentivo à criação e consolidação de intercâmbio e cooperação técnico-científicas e à formação de redes de colaboração;

1.2.7 Consolidação de mecanismos de financiamento, tais como bolsas de pesquisa para recém-doutores, que permitam a incorporação de um maior número de pesquisadores aos grupos em atividade;

1.2.8 Criar mecanismos que garantam a descentralização de investimentos, com especial atenção para o desenvolvimento da capacidade de pesquisa em epidemiologia nas regiões Centro-Oeste e Norte do país, estimulando a inclusão de grupos de pesquisas daquelas regiões em redes nacionais de pesquisas epidemiológicas;

1.2.9 Estimular a constituição de redes entre grupos de pesquisa consolidados, com vistas ao enfrentamento de questões de investigação complexas ou a realização de projetos de grandes dimensões, como grandes estudos longitudinais;

1.2.10 Apoiar a Rede Latino-americana e Caribenha de Epidemiologia - EPILAC, constituída durante o VI Congresso Brasileiro de Epidemiologia;

1.2.11 Promover seminários nacionais para a divulgação de experiências com pesquisas interdisciplinares no campo da Saúde Coletiva; 
1.2.12 Instar para que as programações dos congressos de epidemiologia favoreçam o diálogo interdisciplinar, tanto no interior do campo da Saúde Coletiva como também com disciplinas externas ao campo.

\section{A divulgação científica em Epidemiologia}

\subsection{Problemas identificados}

2.1.1 Poucos meios de divulgação da produção científica em epidemiologia, e longos períodos de tempo entre a submissão e a publicação nos periódicos nacionais;

2.1.2 Incipiente visibilidade da produção nacional em epidemiologia;

2.1.3 Incipiência da publicação de jovens pesquisadores, principalmente dos produtos derivados da pós-graduação acadêmica e profissional;

\subsection{Ações propostas}

2.2.1 Ampliar o número de fascículos por volume nas revistas nacionais da área já consolidadas, viabilizando maior volume de publicações e redução dos prazos de publicação;

2.2.2 Garantir financiamento suficiente para as revistas nacionais da área;

2.2.3 Incentivar a adoção de mecanismos mais eficientes de submissão e avaliação dos artigos, como os procedimentos eletrônicos, visando a redução dos prazos de publicação;

2.2.4 Valorizar o trabalho dos pareceristas, solicitando a divulgação de relatórios anuais que indiquem a produção respectiva de cada um, e que seja possível a incorporação desse registro no currículo Lattes;

2.2.5 Incentivar a adoção da versão eletrônica em inglês, para as revistas da área, especialmente a Revista Brasileira de Epidemiologia;

2.2.6 Tomar as providências necessárias para Indexar a Revista Brasileira de Epidemiologia ao MedLine; 
2.2.7 Quantificar e avaliar os impactos bibliométricos da produção nacional em Epidemiologia;

2.2.8 Desenvolver novos indicadores não bibliométricos do impacto da pesquisa epidemiológica;

2.2.9 Desenvolver formas de registro de produtos tecnológicos (tecnologias leves) oriundos dos trabalhos de conclusão do mestrado profissional e de outras pesquisas, visando dar visibilidade a essa produção e também possibilitar o desenvolvimento de critérios apropriados de avaliação;

2.2.10 Manter as grades de classificação dos trabalhos apresentados aos congressos brasileiros de epidemiologia para permitir o acompanhamento da série histórica da produção em cada temática;

2.2.11 Solicitar aos editores científicos que a comunicação com os autores seja respeitosa e feita no sentido de estimular o aprimoramento dos manuscritos sem afastar os pesquisadores iniciantes;

2.3.12 Estabelecer oficinas de redação científica ou estratégias equivalentes nos cursos de pós-graduação, auxiliando os alunos a tornarem-se autores;

2.3.13 Apoiar a elaboração de um manual eletrônico "Como escrever artigos científicos em epidemiologia” para uso mais ampliado;

2.3.14 Definir perfis mais apropriados de publicação nas revistas dirigidas aos profissionais de serviços, admitindo formatos mais flexíveis de comunicação, de modo a incluir, entre outros, informes técnicos, relatos de experiência, desenvolvimento de tecnologias;

2.3.15 Fortalecer iniciativas como a Expo-Epi para a divulgação de experiências bem sucedidas e produção de conhecimentos sobre os serviços de saúde.

\section{Difusão do conhecimento em Epidemiologia}

\subsection{Problemas identificados}

3.1.1 Precariedade da comunicação social entre pesquisadores e a comunidade; 
3.1.2 Falta de conteúdos curriculares relativos à capacitação no desenvolvimento e aplicação de estratégias de difusão do conhecimento para a população, nos cursos de pós-graduação;

3.1.3 Deficiências na atuação da ABRASCO no que se refere à comunicação científica.

\subsection{Ações propostas}

3.2.1 Necessidade de desenvolver estratégias que facilitem a transferência do conhecimento produzido para a população;

3.2.2 Incluir conteúdos de comunicação social nos cursos de formação em Epidemiologia;

3.2.3 Desenvolver estratégias apropriadas de devolução dos resultados de pesquisa para grupos sociais organizados;

3.2.4 Construir uma "sala de imprensa" no site da ABRASCO, com conteúdo direcionado para o jornalismo científico;

3.2.5 Elaborar consensos nacionais sobre assuntos de interesse da população em geral, para serem veiculados no site da ABRASCO.

\section{4. Ética na pesquisa em epidemiologia}

\subsection{Problemas identificados}

4.1.1 Inadequação de alguns dos procedimentos recomendados nos atuais documentos normativos da CONEP, para o manejo de aspectos da Ética em Pesquisa em estudos epidemiológicos voltados para a Saúde Pública;

4.1.2 Falta de avaliação do trabalho dos Comitês de Ética em Pesquisa das instituições da área, para identificar, de modo sistemático, os problemas mais freqüentes, as soluções encontradas e outros aspectos relevantes para o aprimoramento dos procedimentos recomendados;

4.1.3 Falta de soluções institucionais adequadas para a guarda e o armazenamento de questionários e formulários empregados na pesquisa; 
4.1.4 Insuficientes debates e discussões sobre a Ética em Pesquisa no âmbito da Saúde Pública;

4.1.5 Pequena incorporação de conteúdos de Ética em Pesquisa nos programas de pós-graduação.

\subsection{Ações propostas}

4.2.1 Realização de seminários nacionais para discutir e aprofundar aspectos éticos específicos das pesquisas epidemiológicas, visando recolher subsídios para proposição de aprimoramento ao atual Sistema de Ética em Pesquisa, SISNEP, e os respectivos instrumentos reguladores a serem encaminhados à CONEP;

4.2.2 Análise do trabalho dos CEPs em relação aos procedimentos de avaliação de pesquisas epidemiológicas;

4.2.3 Elaboração de um boletim especial da ABRASCO sobre o tema da Ética em Pesquisa.

\section{Teoria e Metodologia na pesquisa epidemiológica}

\subsection{Problemas identificados}

5.1.1 Pouco amadurecimento na incorporação de modelos teóricos na epidemiologia;

5.1.2 Falta de adequação e uniformização na construção de variáveis que expressam dimensões conceituais complexas, tais como sexo, gênero, raça, etnia, classe social e outras, com vistas à sua utilização em estudos epidemiológicos;

5.1.3 Desafios para a adequação das respostas da epidemiologia à dupla demanda, i.e., necessidades científicas e políticas;

5.1.4 Insuficiência das temáticas abordadas na pesquisa epidemiológica, para dar conta dos desafios da prática dos serviços e das necessidades de saúde da população. 


\subsection{Ações propostas}

5.2.1 Realizar eventos com o objetivo de ampliar, confrontar visões e perspectivas, bem como aprimorar a elaboração de modelos teóricos, e incentivar o seu efetivo uso na pesquisa epidemiológica;

5.2.2 Promover discussões sobre métodos relacionados à construção de variáveis que representem, de forma mais satisfatória, conceitos complexos tais como sexo, gênero, raça, etnia, classe social e outros, com vistas à sua utilização em estudos epidemiológicos;

5.2.3 Incentivo ao desenvolvimento de metodologias e pesquisas para a avaliação da incorporação do conhecimento epidemiológico nas políticas públicas, e do seu resultante impacto político;

5.2.4 Continuar o apoio à realização de oficinas em torno de temas relevantes para a Saúde Pública, com a participação de gestores, profissionais da saúde, usuários e pesquisadores, de modo a propiciar maior intercâmbio entre serviços e academia. 